\title{
Near Linear Lower Bound for Dimension Reduction in $\ell_{1}$
}

\author{
Alexandr Andoni \\ Microsoft Research SVC \\ Mountain View, CA, USA \\ Email:andoni@mit.edu
}

\author{
Moses S. Charikar, Ofer Neiman, Huy L. Nguyen \\ Department of Computer Science \\ Center for Computational Intractability \\ Princeton University, Princeton, NJ, USA \\ Email: $\{$ moses, oneiman, hlnguyen $\} @$ cs.princeton.edu
}

\begin{abstract}
Given a set of $n$ points in $\ell_{1}$, how many dimensions are needed to represent all pairwise distances within a specific distortion? This dimension-distortion tradeoff question is well understood for the $\ell_{2}$ norm, where $O\left((\log n) / \epsilon^{2}\right)$ dimensions suffice to achieve $1+\epsilon$ distortion. In sharp contrast, there is a significant gap between upper and lower bounds for dimension reduction in $\ell_{1}$. A recent result shows that distortion $1+\epsilon$ can be achieved with $n / \epsilon^{2}$ dimensions. On the other hand, the only lower bounds known are that distortion $\delta$ requires $n^{\Omega\left(1 / \delta^{2}\right)}$ dimensions and that distortion $1+\epsilon$ requires $n^{1 / 2-O(\epsilon \log (1 / \epsilon))}$ dimensions. In this work, we show the first near linear lower bounds for dimension reduction in $\ell_{1}$. In particular, we show that $1+\epsilon$ distortion requires at least $n^{1-O(1 / \log (1 / \epsilon))}$ dimensions.
\end{abstract}

Our proofs are combinatorial, but inspired by linear programming. In fact, our techniques lead to a simple combinatorial argument that is equivalent to the LP based proof of BrinkmanCharikar for lower bounds on dimension reduction in $\ell_{1}$.

Keywords-dimension reduction, metric embedding

\section{INTRODUCTION}

In this paper, we study dimension reduction questions of the following form: Given a set $X$ of $n$ points in $\mathbb{R}^{d}$ with distances measured by the $\ell_{p}$ norm, the objective is to find an embedding $f: X \rightarrow \mathbb{R}^{d^{\prime}}$ with $d^{\prime} \ll d$ that roughly preserves pairwise distances. We say that the distortion of the embedding is $\delta$ if there exists a constant $c$ such that for all $x, y \in X$ :

$$
c|| x-y\left\|_{p} \leq\right\| f(x)-f(y)\left\|_{p} \leq c \cdot \delta\right\| x-y \|_{p}
$$

Dimension reduction has received a lot of attention in recent years, due to its numerous applications in computer science (see [8], [9], [11], [12], [7] and the references therein). Furthermore, it is a topic of interest from a purely mathematical point of view and has been well-studied in the area of functional analysis.

For the $\|\cdot\|_{2}$ norm, the famous Johnson-Lindenstrauss Lemma [10] states that for any $\epsilon>0$ one can linearly embed an $n$ point subset of $\ell_{2}$ into $d^{\prime}=O\left((\log n) / \epsilon^{2}\right)$ dimensions with distortion at most $1+\epsilon$. This is essentially tight, as shown by Alon [1]. Unfortunately, the situation for spaces other than Euclidean is far from understood. In this work, we focus on the $\ell_{1}$ norm for which there are huge gaps between currently known upper and lower bounds.
Ball [2] showed that in order to embed some $n$ point subset of $\ell_{1}$ isometrically (with distortion 1 ), $\left(\begin{array}{l}n \\ 2\end{array}\right)$ dimensions are necessary (and always suffice). A strong lower bound for dimension reduction in $\ell_{1}$ was shown by Brinkman and Charikar [4], with a simpler geometric proof by Lee and Naor [13]. They exhibit an $n$ point subset of $\ell_{1}$ requiring dimension at least $n^{\Omega\left(1 / D^{2}\right)}$ for distortion $D$. They also showed that for distortion $1+\epsilon$ the dimension required is at least $n^{1 / 2-\tilde{O}(\epsilon)}$. There has been some progress on upper bounds: Indyk [8] showed a weak form of dimension reduction in $\ell_{1}$, which is already sufficient for certain applications such as norm estimation in streaming, and approximate nearest neighbor search (see, for example, Datar et al. [6]). For dimension reduction with $1+\epsilon$ distortion, Schechtman [15], and Talagrand [16] showed that $O\left((n \log n) / \epsilon^{2}\right)$ dimensions suffice. This was recently improved by Newman and Rabinovich [14] to $O\left(n / \epsilon^{2}\right)$ based on the sparsification techniques of Batson, Spielman, and Srivastava [3].

In the $1+\epsilon$ distortion regime, the gap between the best known lower and upper bounds is $\Omega\left(n^{1 / 2}\right)$. We narrow this gap substantially, and show that when the distortion is close to 1 , the required number of dimensions is near linear.

Theorem 1. For any $\epsilon>0$ and integer $n>0$ there exists an $n$ point subset $X$ of $\ell_{1}$, such that any embedding of $X$ into $\ell_{1}$ with distortion $1+\epsilon$ requires dimension at least $n^{1-O(1 / \log (1 / \epsilon))}$.

\subsection{Techniques}

The lower bound of [4] and [13] was obtained by using the so-called diamond graph, which is defined recursively: $G_{0}$ is a single edge, and $G_{i}$ is obtained from $G_{i-1}$ by replacing every edge with a 4-cycle where two antipodal vertices of the cycle are identified with the original end points of the edge. [4] established the bound by exhibiting a dual solution of a certain linear program that captures the constraints imposed on pairwise distances by a line embedding. The alternate, simpler proof of [13] first showed a lower bound on embedding the diamond graph in $\ell_{p}$ for $p$ close to 1 , and then used the relation between $\ell_{p}$ and $\ell_{1}$ norms in order to achieve the dimension lower bound for embedding into $\ell_{1}$. The metric based on the diamond graph in these lower 
bounds is isometrically embeddable in $\sqrt{n}$ dimensions hence it cannot establish a lower bound better than $\sqrt{n}$.

The basis for our near linear lower bound is a seriesparallel graph that generalizes the diamond graph: in the recursive construction, edges are replaced by cycles of length $2 / \epsilon-$ we call this the recursive cycle graph. Similarly to [5], [4], we use the notion of stretch (defined in the sequel) to argue about the number of dimensions needed for a low distortion $\ell_{1}$ embedding. Informally, a stretch $s$ embedding is a distribution over line embeddings where no pairwise distance is increased by a factor of more than $s$. We show that a Poincaré inequality - a linear inequality (involving distances for antipodal pairs of the cycles in the graph and edges) - must hold for all embeddings of limited stretch but cannot hold for any low distortion embedding. This implies that any low distortion embedding must have high stretch, and hence, high dimension.

While the use of stretch for proving dimension lower bounds has been established in previous work, using this proof technique involves the following two steps: (1) identifying an appropriate Poincaré inequality, and (2) proving that it holds for any line embedding with low stretch. Both steps are challenging in general and this is our main technical contribution. We made crucial use of linear programming in executing these two steps.

We briefly elaborate on this methodology for proving dimension lower bounds. In order to capture the limitations of line embeddings with low stretch, we write (1) linear constraints on pairwise distances (viewed as variables) using the fact that they arise from a line embedding and (2) constraints imposed by the stretch $\leq s$ condition. In general, there is no principled way to write down the appropriate constraints (1) for any line embedding. A key idea we use in expressing these constraints is to incorporate information about the line embedding by using a convenient renaming of vertices which is a function of the line embedding. Next, we write (3) a set of constraints on average distances on certain groups of pairwise distances implied by the distortion $\leq \delta$ condition, using the intuition about the particular metric under consideration, that low stretch embeddings tend to expand certain groups of distances, and contract other groups of distances. For the lower bound example in this paper, these groups are the set of edges and for every level, the set of antipodal pairs at that level. The infeasibility of this system of linear inequalities leads to a proof that no stretch $s$ embedding can have distortion $\leq \delta$. In fact the Poincaré inequality that establishes this is obtained from a linear combination of the constraints (3) where the multipliers are the values of the dual variables for these constraints.

This is in fact how we derived the inequality we use to establish our dimension lower bound. Although the proof we present here makes no reference to linear programming, it is in fact a combinatorial interpretation of the LP based proof (and more insightful than an LP duality based proof, we believe). Our proof uses a certain charging argument and inspired by this, we give a simple combinatorial proof of the original result of [4] that is equivalent to the original LP based proof. The Poincaré-type inequality and the distances involved are similar to the case of the recursive cycle graph but with a slight twist: some of the distances are directed distances i.e. they could take positive and negative values.

\subsection{Organization}

In Section 2 we recall the notion of stretch and its connection to distortion and dimension. In Section 3.2 we demonstrate our techniques in a relatively simple setting, and prove Theorem 2, a lower bound for $C_{n}$, the cycle graph on $n$ vertices. Then in Section 3.3 we extend the methods to the recursive cycle graph, and prove Theorem 1. Finally in Section 4 we show the versatility of our technique, and reprove [4] lower bound by a somewhat simpler argument.

\section{Preliminaries}

Definition 1. An embedding of a metric $(X, d)$ to $\ell_{1}$ with stretch $s$ and distortion $D$ is a distribution over mappings $f: X \rightarrow \mathbb{R}$ that satisfies the following conditions for all $x, y \in X$,

1) stretch constraint: $|f(x)-f(y)| \leq s \cdot d(x, y)$ for all $f$ in the support of the distribution.

2) distortion constraint:

$$
d(x, y) / D \leq \mathbb{E}_{f}[|f(x)-f(y)|] \leq d(x, y)
$$

The following claim relating stretch to dimension was shown in [5]

Claim 1. If a metric space $(X, d)$ has an embedding into $\ell_{1}^{s}$ with distortion $\alpha$, then it also has embedding with distortion $\alpha$ and stretch at most $s$.

In order to show the tradeoff between stretch and distortion, we will prove certain Poincaré-type inequalities and apply the following lemma, which essentially was proven in [5]. A proof is provided below for completeness.

Lemma 2. Consider a metric $(X, d)$. If there are positive numbers $\alpha_{x, y}, \beta_{x, y}$ and $\gamma$ such that for any line embedding $f: X \rightarrow \mathbb{R}$ of stretch at most $s$,

$$
\sum_{x, y \in X} \beta_{x, y}|f(x)-f(y)|-\sum_{x, y \in X} \alpha_{x, y}|f(x)-f(y)| \leq \gamma
$$

then any embedding to $\ell_{1}$ of stretch at most $s$ has distortion at least $\frac{\sum_{x, y} \beta_{x, y} d(x, y)}{\sum_{x, y} \alpha_{x, y} d(x, y)+\gamma}$.

Proof: Consider an embedding, i.e. a distribution over line embeddings $f$, with stretch $s$ and distortion $D$. By definition,

$$
\sum_{x, y \in X} \alpha_{x, y} \mathbb{E}_{f}[|f(x)-f(y)|]+\gamma \leq \sum_{x, y \in X} \alpha_{x, y} d(x, y)+\gamma
$$


and

$$
\sum_{x, y \in X} \beta_{x, y} \mathbb{E}_{f}[|f(x)-f(y)|] \geq \sum_{x, y \in X} \beta_{x, y} d(x, y) / D
$$

Since inequality (1) holds for all line embeddings, we have

$$
\sum_{x, y \in X} \beta_{x, y} \mathbb{E}_{f}[|f(x)-f(y)|] \leq \sum_{x, y \in X} \alpha_{x, y} \mathbb{E}_{f}[|f(x)-f(y)|]+\gamma
$$

Combining the above inequalities, we get $\frac{\sum_{x, y} \beta_{x, y} d(x, y)}{D} \leq$ $\sum_{x, y} \alpha_{x, y} d(x, y)+\gamma$ i.e. $D \geq \frac{\sum_{x, y} \beta_{x, y} d(x, y)}{\sum_{x, y} \alpha_{x, y} d(x, y)+\gamma}$.

We note that, like in the setting of embeddability into $\ell_{1}$ (without dimension restriction), if a metric $X$ does not admit an embedding of stretch $s$ for a certain distortion, then there exists a proof of non-embeddability of the above form (that is, there exist $\alpha$ and $\beta$ satisfying the condition of Lemma 2).

Notation 1. Let $x \circ y$ denote the concatenation of two strings $x$ and $y$.

\section{THE LOWER BOUND}

\subsection{Proof Overview}

Our lower bound is based on the shortest path metric of a recursively constructed graph. The basic ingredient of the construction is the cycle $C_{2 k}$ and we first establish a dimension-distortion tradeoff for this simple graph. The intuition for the lower bound comes from the basic case of the Borsuk-Ulam theorem: in any continuous embedding of the cycle into $\mathbb{R}$, an antipodal pair is mapped to the same point. This intuition is made precise in a discrete setting by the notion of flipped antipodals (see Definition 2). We establish an inequality relating antipodal distances that "tend to contract" to edge distances that "tend to expand" in a line embedding. The proof then uses a charging argument, based on the flipped antipodals and the triangle inequality, to bound the lengths of the antipodals by the lengths of the edges. Finally the stretch bound is used on some edges that were charged "often".

$C_{2 k}$ has an isometric embedding with stretch $k$. Our proof shows that any distortion $1+\epsilon$ embedding of $C_{2 k}$ must have stretch at least $\Omega(1 / \epsilon)$. This lower bound on stretch is tight up to constants, since $C_{2 k}$ has a $1+\epsilon$ distortion embedding with stretch $O(1 / \epsilon)$.

Next we consider a certain product construction of the cycle, that we call the recursive cycle graph $G_{t}-$ this has $(2 k)^{t}$ edges and $\Theta\left((2 k)^{t}\right)$ vertices. For this product construction, we show (roughly speaking) that the stretch bound is raised to the power $t$. In particular, we show that any $1+1 / k$ distortion embedding of $G_{t}$ must have stretch $(\Omega(k))^{t}$ by reasoning about the lengths of edges and antipodal pairs. This gives our main result showing a near linear lower bound for $1+\epsilon$ distortion embeddings of $\ell_{1}$. The lower bound we show for $G_{t}$ is almost optimal in the following sense: $G_{t}$ has an embedding with stretch $k^{t}$ that is isometric for all edges and antipodal pairs. Also, there is a stretch $(O(1 / \epsilon))^{t}$ embedding of $G_{t}$ that has distortion at most $1+\epsilon$ for edges and antipodal pairs.

\subsection{Simple Case}

We begin by proving the following theorem for the shortest path metric of the cycle $C_{2 k}$. In this process, we will establish some machinery that will be useful for the general case later.

Theorem 2. For any integer $k$ and $\epsilon \geq 1 / k$, any embedding of $C_{2 k}$ into $\ell_{1}$ with distortion at most $1+\epsilon$ requires dimension at least $\Omega(1 / \epsilon)$.

Assume w.l.o.g that $k$ is even, and label the vertices of $C_{2 k}$ by $a_{1}, a_{2}, \ldots, a_{k}, a_{-1}, a_{-2}, \ldots, a_{-k}$, so that for every $1 \leq i \leq k,\left(a_{i}, a_{-i}\right)$ is an antipodal pair denoted by $d_{i}$. In what follows we identify indices $k+i$ with $-i$ and $-(k+i)$ with $i$ for any $1 \leq i \leq k$. Fix some $f: C_{2 k} \rightarrow \mathbb{R}$, and let $L=\sum_{i=1}^{k}\left|f\left(a_{i}\right)-f\left(a_{-i}\right)\right|$ and $E=\sum_{i=1}^{k} \mid f\left(a_{i}\right)-$ $f\left(a_{i+1}\right)|+| f\left(a_{-i}\right)-f\left(a_{-(i+1)}\right) \mid$.

Lemma 3. Let $f: C_{2 k} \rightarrow \mathbb{R}$ be a line embedding with stretch at most $s$, then the following Poincaré-type inequality holds

$$
2 L-k(1-2 \epsilon) E \leq s \cdot(2 \epsilon k)^{2}
$$

Before proving this lemma, let us show that it implies Theorem 2. Apply Lemma 2 with the appropriate coefficients $\alpha_{a_{i}, a_{i+1}}=\alpha_{a_{-i}, a_{-(i+1)}}=k(1-2 \epsilon), \beta_{a_{i}, a_{-i}}=2$ for all $1 \leq i \leq k$ (set $\alpha$ and $\beta$ to zero everywhere else) and $\gamma=s \cdot(2 \epsilon k)^{2}$, to conclude that any embedding $F: C_{2 k} \rightarrow \ell_{1}$ with stretch at most $s$ must have distortion at least

$$
\begin{aligned}
\frac{\sum_{x, y \in V\left(C_{2 k}\right)} \beta_{x, y} d(x, y)}{\sum_{x, y \in V\left(C_{2 k}\right)} \alpha_{x, y} d(x, y)+\gamma} & =\frac{2 k^{2}}{2 k^{2}(1-2 \epsilon)+s \cdot(2 \epsilon k)^{2}} \\
& =\frac{1}{1-2 \epsilon+2 s \epsilon^{2}}
\end{aligned}
$$

where it was used that the total antipodal length in $C_{2 k}$ is $k^{2}$ and the total edge length is $2 k$. Finally set $s=1 /(2 \epsilon)$ and obtain distortion at least $\frac{1}{1-2 \epsilon+\epsilon}>1+\epsilon$. By Claim 1 this suggests that an embedding into fewer than $s=1 /(2 \epsilon)$ dimensions cannot have distortion bounded by $1+\epsilon$, so it concludes the proof of Theorem 2. We now turn to prove the main lemma.

\section{Proof of Lemma 3:}

The notion of flipped antipodal pairs is key to showing that some antipodal distances tend to be short relative to distances of edges in any line embedding.

Definition 2. Let $f: C_{2 k} \rightarrow \mathbb{R}$ and fix $1 \leq i \leq k$. Two antipodal pairs $d_{i}, d_{i+1}$ are called flipped under $f$ if $\operatorname{sign}\left(f\left(a_{i}\right)-f\left(a_{-i}\right)\right) \neq \operatorname{sign}\left(f\left(a_{i+1}\right)-f\left(a_{-(i+1)}\right)\right)$ (or if $\left.\operatorname{sign}\left(f\left(a_{i}\right)-f\left(a_{-i}\right)\right)=0\right)$. 
Claim 4. In any map $f: C_{2 k} \rightarrow \mathbb{R}$ there exist flipped antipodal pairs $d_{i}, d_{i+1}$, which satisfy:

$$
\begin{aligned}
& \left|f\left(a_{i}\right)-f\left(a_{i+1}\right)\right|+\left|f\left(a_{-i}\right)-f\left(a_{-(i+1)}\right)\right| \\
\geq & \left|f\left(a_{i}\right)-f\left(a_{-i}\right)\right|+\left|f\left(a_{i+1}\right)-f\left(a_{-(i+1)}\right)\right|
\end{aligned}
$$

Proof: To see the existence of the flipped antipodal pairs, consider the values $f\left(a_{1}\right)-f\left(a_{-1}\right), f\left(a_{2}\right)-$ $f\left(a_{-2}\right), \ldots, f\left(a_{k}\right)-f\left(a_{-k}\right), f\left(a_{-1}\right)-f\left(a_{1}\right)$. Since the first is the opposite of the last, the sign must change at some point, and there will be the flipped pair.

To prove (3), assume w.l.o.g that $f\left(a_{i}\right) \geq f\left(a_{-i}\right)$ and $f\left(a_{i+1}\right) \leq f\left(a_{-(i+1)}\right)$. Then

$$
\begin{aligned}
& \left(f\left(a_{i}\right)-f\left(a_{-i}\right)\right)+\left(f\left(a_{-(i+1)}\right)-f\left(a_{i+1}\right)\right) \\
& =\left(f\left(a_{i}\right)-f\left(a_{i+1}\right)\right)+\left(f\left(a_{-(i+1)}\right)-f\left(a_{-i}\right)\right) \\
& \leq\left|f\left(a_{i}\right)-f\left(a_{i+1}\right)\right|+\left|f\left(a_{-(i+1)}\right)-f\left(a_{-i}\right)\right| .
\end{aligned}
$$

Labeling Cycle Edges: Consider the two flipped antipodal pairs $d_{i}, d_{i+1}$ guaranteed to exist by Claim 4 . Let $e_{0}$ be the length under $f$ of the edge connecting $a_{i}$ to $a_{i+1}$, i.e. $e_{0}=\left|f\left(a_{i}\right)-f\left(a_{i+1}\right)\right|$, and $e_{0^{\prime}}=\left|f\left(a_{-i}\right)-f\left(a_{-(i+1)}\right)\right|$. Similarly, let $e_{j}, e_{-j}, e_{j^{\prime}}$ and $e_{-j^{\prime}}$ be the length under $f$ of the four edges of distance $j$ from $e_{0}, e_{0^{\prime}}$ respectively, for $j=1, \ldots, k / 2$, see Figure 1 (note that the last two edges have multiple names under this labeling, but will not be considered in our proof). Furthermore, let $E_{j}=e_{j}+e_{-j}+e_{j^{\prime}}+e_{-j^{\prime}}$ (for ease of notation we define $\left.e_{-0}=e_{-0^{\prime}}=0\right)$. We also abuse notation and write $d_{j}=\left|f\left(a_{j}\right)-f\left(a_{-j}\right)\right|$.

By (3) we have that

$$
d_{i}+d_{i+1} \leq e_{0}+e_{0^{\prime}}=E_{0} .
$$

Consider now the two adjacent antipodals $d_{i-1}$ and $d_{i+2}$. By the triangle inequality

$d_{i-1}+d_{i+2} \leq\left(e_{-1}+d_{i}+e_{1^{\prime}}\right)+\left(e_{1}+d_{i+1}+e_{-1^{\prime}}\right) \leq E_{0}+E_{1}$.

In a similar manner for any integer $0 \leq j<k / 2$ we bound

$$
\begin{aligned}
d_{i-j}+d_{i+1+j} & \leq d_{i}+d_{i+1}+\sum_{h=1}^{j}\left(e_{j}+e_{j^{\prime}}+e_{-j}+e_{-j^{\prime}}\right) \\
& \leq E_{0}+E_{1}+\cdots+E_{j} .
\end{aligned}
$$

So we have the following antipodal charging inequality (see Figure 2):

$$
2 L \leq 2 \sum_{j=0}^{k / 2-1}\left(E_{0}+\cdots+E_{j}\right)=\sum_{j=0}^{k / 2-1}(k-2 j) E_{j} .
$$

For simplicity assume that $\epsilon=q / k$ for some integer $q \geq 1$, then

$$
\begin{aligned}
2 L-k(1-2 \epsilon) E & \leq \sum_{j=0}^{k / 2-1}(k-2 j) E_{j}-\sum_{j=0}^{k / 2-1}(k-2 q) E_{j} \\
& \leq 2 \sum_{j=0}^{q-1}(q-j) E_{j} .
\end{aligned}
$$

Since $f$ has stretch at most $s, E_{0} \leq 2 s$ and $E_{j} \leq 4 s$ for $j \geq 1$. Hence,

$$
\begin{aligned}
2 L-k(1-2 \epsilon) E & \leq 4 s q+8 s \sum_{j=1}^{q-1}(q-j) \\
& =4 s q+8 s(q-1) q / 2 \\
& =s \cdot(2 \epsilon k)^{2},
\end{aligned}
$$

which concludes the proof of the lemma.

\subsection{Recursive Cycle Graph}

In this section we extend our techniques to prove Theorem 1. The bulk of the proof is showing that the recursive cycle graph requires high dimension for small distortion, then in Section 3.4 we exhibit a subset of $n$ points in $\ell_{1}$ that has the same dimension-distortion tradeoff. Let $k=1 / \epsilon$, and w.l.o.g assume that $k$ is an even integer. The graph is defined recursively as follows: $G_{0}$ is a single edge, and for $i>0, G_{i}$ is obtained from $G_{i-1}$ by replacing every edge $(u, v) \in E\left(G_{i-1}\right)$ with a disjoint copy of $C_{2 k}$, where some antipodal pair, say $a_{1}$ and $a_{-1}$, are associated with $u, v$ respectively, see Figure 3 . The edge $(u, v)$ is called the parent edge for the cycle it induces. We call $G_{t}$ the level $t$ graph, note that the number of edges in $G_{t}$ is $(2 k)^{t}$, the number of vertices is $n=\Theta\left((2 k)^{t}\right)$ and the diameter is $k^{t} \approx n^{1-1 / \log k}$.

Fix some $f: G_{t} \rightarrow \mathbb{R}$. In what follows we define a labeling scheme for the edges and antipodals of $G_{t}$ (note that this labeling depends on $f$ ), for ease of presentation, the label of a pair will also indicate its length under $f$. For $1 \leq i \leq t$, let $\mathcal{C}_{i}$ be the collection of level $i$ cycles (note that $\left|\mathcal{C}_{i}\right|=$ $\left.(2 k)^{i-1}\right)$. Let $A=\left\{-k / 2, \ldots, k / 2,(-k / 2)^{\prime}, \ldots,(k / 2)^{\prime}\right\}$ be a set of indices. The single edge of $G_{0}$ is labeled $e_{\emptyset}$, and the rest of the edges and antipodals are labeled recursively. For level $i>0$, fix some $C \in \mathcal{C}_{i}$ whose parent is an edge labeled $e_{x}$ for $x \in A^{i-1}$. Recall that by Claim $4 C$ will have a pair of flipped antipodals under $f$. An edge in $C$ is labeled by $e_{x \circ z}$ where $z \in A$ chosen by the same labeling for the single cycle case, which is determined by the flipped antipodals, and is depicted in Figure 1. The flipped antipodals will be named $d_{x \circ 0}, d_{x \circ 0^{\prime}}$, and the two antipodals of distance $j$ from the flipped ones will be named $d_{x \circ j}, d_{x \circ j^{\prime}}$ (for notational convenience we let $d_{x \circ-j}=d_{x \circ-j^{\prime}}=0$ and ignore the multiple labeled edges, as these are never charged). 


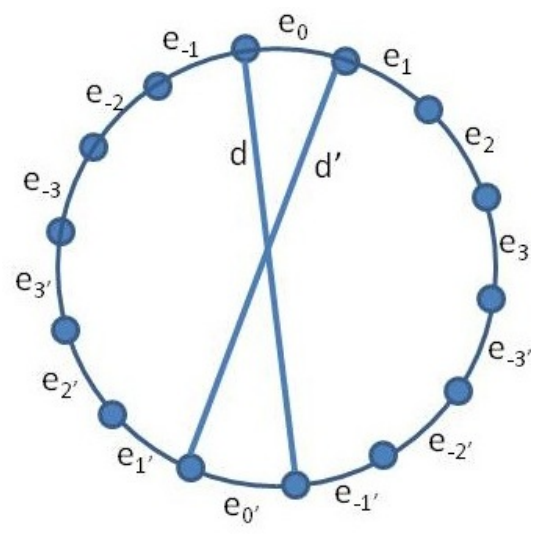

Figure 1. Notation for cycle edges, $d$ and $d^{\prime}$ are the flipped antipodals
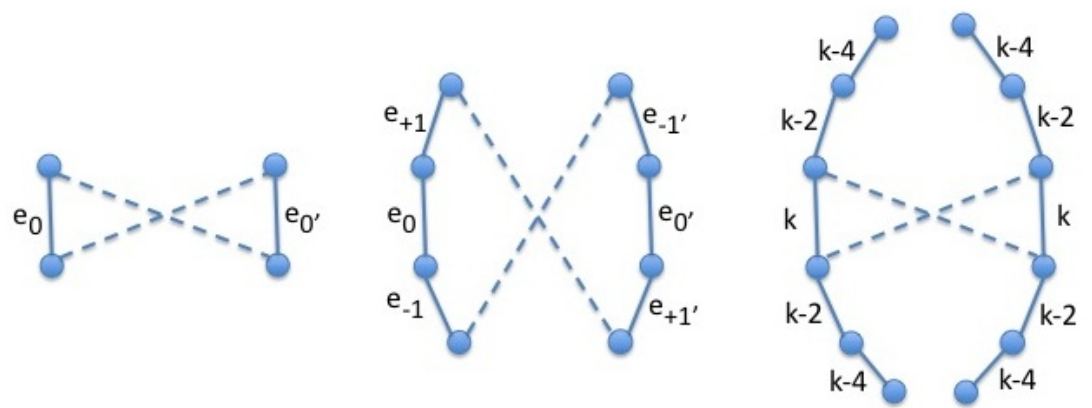

Figure 2. Antipodal charging scheme: in two left images the antipodals are charged to the depicted edges, right image shows the total edge charge (the dashed lines are the flipped antipodals)

Let $L_{i}=\sum_{x \in A^{i}} d_{x}$ be the total antipodal length in level $i$, and $E=\sum_{x \in A^{t}} e_{x}$ the total (level $t$ ) edge length.

Lemma 5. Let $f: G_{t} \rightarrow \mathbb{R}$ be a line embedding with stretch at most $s$, then the following Poincaré-type inequality holds

$$
\sum_{i=1}^{t} 2^{t+1-i} L_{i}-(t k-2 t) E \leq s \cdot t(32)^{t} .
$$

Once again, before attempting to prove this lemma, we show that it implies Theorem 1. Apply Lemma 2 with the appropriate coefficients: for $x \in A^{t}$ let $\alpha_{e_{x}}=t k-2 t$, and for all $1 \leq i \leq t$ and $x \in A^{i}$ let $\beta_{d_{x}}=2^{t+1-i}$ (set $\alpha$ and $\beta$ to zero everywhere else) and $\gamma=s \cdot t(32)^{t}$. Note that there are $(2 k)^{i-1} \cdot k$ antipodals in level $i$, each of length $k^{t+1-i}$, so the total antipodal length in level $i$ is $2^{i-1} k^{t+1}$, and the total edge length is $(2 k)^{t}$. Now, any embedding $F: G_{t} \rightarrow \ell_{1}$ with stretch at most $s$ must have distortion at least

$$
\begin{aligned}
& \frac{\sum_{x, y \in V\left(G_{t}\right)} \beta_{x, y} d(x, y)}{\sum_{x, y \in V\left(G_{t}\right)} \alpha_{x, y} d(x, y)+\gamma}=\frac{\sum_{i=1}^{t} 2^{t+1-i} \cdot 2^{i-1} k^{t+1}}{(t k-2 t)(2 k)^{t}+s \cdot t(32)^{t}} \\
& =\frac{t 2^{t} k^{t+1}}{t 2^{t} k^{t+1}-2 t(2 k)^{t}+s \cdot t(32)^{t}}
\end{aligned}
$$

Finally set $s=(k / 16)^{t}$ and obtain distortion at least

$$
\frac{t 2^{t} k^{t+1}}{t 2^{t} k^{t+1}-2 t(2 k)^{t}+t(2 k)^{t}}=\frac{1}{1-1 / k}>1+1 / k .
$$

By Claim 1 this suggests that an embedding into fewer than $(\Omega(k))^{t}=n^{1-O(1 / \log k)}$ dimensions cannot have distortion bounded by $1+1 / k$ (recall that $\epsilon=1 / k$ ). It remains to prove the main lemma.

\section{Proof of Lemma 5:}

As the inequality (5) suggests, we will charge antipodal lengths from all levels to the level $t$ edges. The following claim will be useful.

Claim 6. Let $e_{x}$ be the length of a level $i$ edge in $G_{t}$ for some $1 \leq i \leq t$ and $x \in A^{i}$. Let $E\left(e_{x}\right)=\sum_{y \in A^{t-i}} e_{x \circ y}$ (this is the total length of the level t edges whose ancestor is $e_{x}$ ), then

$$
e_{x} \leq \frac{E\left(e_{x}\right)}{2^{t-i}}
$$

Proof: By inverse induction on $i$. When $i=t$ this is immediate as $E\left(e_{x}\right)=e_{x}$. Assume it holds for $i+1$, and note that the triangle inequality suggests that $e_{x} \leq$ $\frac{1}{2} \sum_{z \in A} e_{x \circ z}$ (in words, the length of an antipodal is at most half of the sum of all edges in a cycle). Now apply the induction hypothesis on each $e_{x \circ z}$, and note that $E\left(e_{x}\right)=\sum_{z \in A} E\left(e_{x \circ z}\right)$ to obtain

$$
e_{x} \leq \frac{1}{2} \sum_{z \in A} e_{x \circ z} \leq \frac{1}{2} \sum_{z \in A} \frac{E\left(e_{x \circ z}\right)}{2^{t-(i+1)}}=\frac{E\left(e_{x}\right)}{2^{t-i}} .
$$




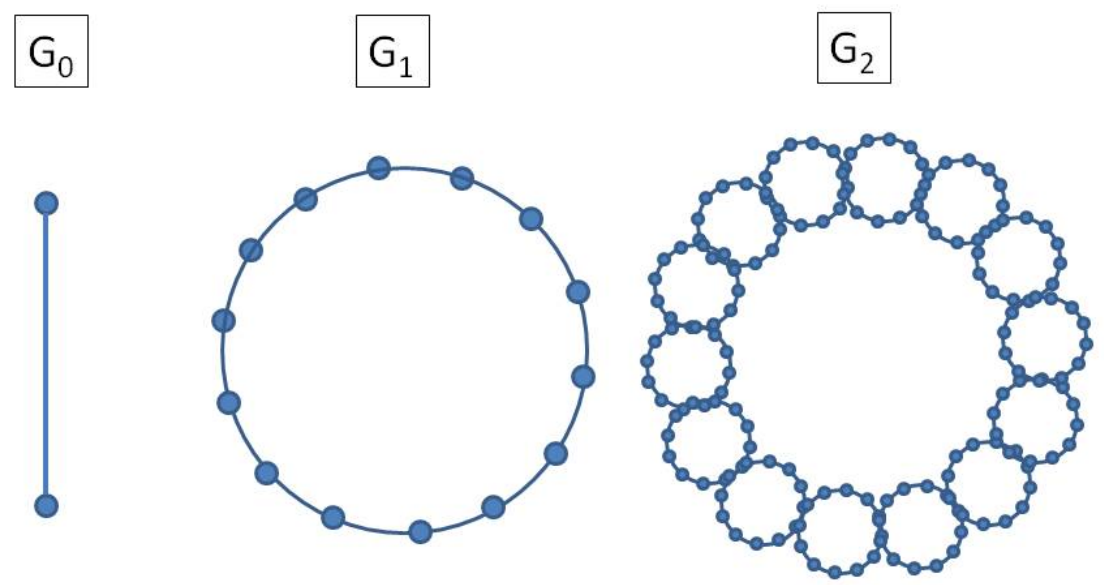

Figure 3. Recursive cycle graph, the first three levels

Fix some $i>0$. By the antipodal charging inequality (4), for any $x \in A^{i-1}$

$$
\begin{aligned}
& 2^{t+1-i} \sum_{z \in A} d_{x \circ z} \\
& \leq 2^{t-i} \sum_{j=0}^{k / 2-1}(k-2 j)\left(e_{x \circ j}+e_{x \circ j^{\prime}}+e_{x \circ-j}+e_{x \circ-j^{\prime}}\right) \\
& \leq \sum_{y \in A^{t-i}} \sum_{j=0}^{k / 2-1} \\
& (k-2 j)\left(e_{x \circ j \circ y}+e_{x \circ j^{\prime} \circ y}+e_{x \circ-j \circ y}+e_{x \circ-j^{\prime} \circ y}\right),
\end{aligned}
$$

where the second inequality is by Claim 6 (for notational convenience we let $e_{x \circ-0 \circ y}=e_{x \circ-0^{\prime} \circ y}=0$ for any $x, y$ ). Now,

$$
\begin{aligned}
2^{t+1-i} L_{i} & =2^{t+1-i} \sum_{x \in A^{i-1}} \sum_{z \in A} d_{x \circ z} \\
& \leq \sum_{x \in A^{i-1}} \sum_{y \in A^{t-i}} \sum_{j=0}^{k / 2-1} \\
& (k-2 j)\left(e_{x \circ j \circ y}+e_{x \circ j^{\prime} \circ y}+e_{x \circ-j \circ y}+e_{x \circ-j^{\prime} \circ y}\right) .
\end{aligned}
$$

Next we attempt to bound $\sum_{i=1}^{t} 2^{t+1-i} L_{i}$. Consider $x \in$ $A^{t}$ and an edge labeled $e_{x}$. The number of times that this edge length was charged by $L_{i}$ in (6) depends only on the symbol $x_{i}$. For $z \in A$ let $|z|$ be the value of $z$, which is simply the number (without the minus or prime signs), and for a vector $x \in A^{t},|x|=\sum_{i=1}^{t}\left|x_{i}\right|$. Now (6) suggests that if $\left|x_{i}\right|=j$ this adds $k-2 j$ to the charge of $e_{x}$. We conclude that the total number of times $e_{x}$ appears is exactly $\sum_{i=1}^{t}\left(k-2\left|x_{i}\right|\right)=t k-2|x|$, in other words,

$\sum_{i=1}^{t} 2^{t+1-i} L_{i} \leq \sum_{x \in A^{t}}(t k-2|x|) e_{x}=t k E-2 \sum_{x \in A^{t}}|x| e_{x}$.
Plugging this into (5) it remains to show that

$$
2 \sum_{x \in A^{t}}(t-|x|) e_{x} \leq s \cdot t(32)^{t} .
$$

Notice that $0 \leq|x| \leq t k / 2$, so the only edges that have a positive sign on the LHS of the inequality are those edges for which $|x|<t$, and these are the edge on which we will apply the stretch assumption, that for any edge $e_{x} \leq s$. Write

$$
\begin{aligned}
2 \sum_{x \in A^{t}}(t-|x|) e_{x} & \leq 2 \sum_{j=0}^{t-1} \sum_{x \in A^{t}:|x|=j}(t-j) e_{x} \\
& \leq 2 t s \sum_{j=0}^{t-1} \sum_{x \in A^{t}:|x|=j} 1,
\end{aligned}
$$

and now we are left with a counting task. For each $1 \leq i \leq t$ fix some value $v_{i} \in\{0,1, \ldots, k / 2\}$, and notice that there are at most $4^{t}$ vectors $x \in A^{t}$ for which $\left|x_{i}\right|=v_{i}$ for all $1 \leq i \leq t$. The number of choices for the $v_{i}$ that sum exactly to $j$, is at most the number of ways of writing $j$ as the sum of $t$ non-negative integers, which is bounded by $2^{j+t}$ (because there are only $t$ possible terms). It follows that

$$
\sum_{j=0}^{t-1} \sum_{x \in A^{t}:|x|=j} 1 \leq t\left(4^{t}\right)^{2}=t 16^{t}
$$

and finally that

$$
2 \sum_{x \in A^{t}}(t-|x|) e_{x} \leq 2 t s \cdot t 16^{t} \leq s \cdot t(32)^{t},
$$

where we used that $2 t \leq 2^{t}$. This concludes the proof of (7), and thus the proof of the lemma.

\subsection{Canonical $\ell_{1}$ Embedding of Recursive Cycle Graph}

Unfortunately, the metric induced by the recursive cycle graph is not necessarily an $\ell_{1}$ metric. Since we are interested 
in the low distortion regime, a bi-Lipschitz map of $G_{t}$ into $\ell_{1}$ will not suffice, so we would like to argue that there exists an $\ell_{1}$ metric with the same "edges" and "antipodals" as the recursive cycle graph. This will suffice to prove the lower bound, because these are the only distances that appeared in the Poincaré-type inequality.

Definition 3. For an integer $t \geq 1$, an embedding $g_{t}: G_{t} \rightarrow$ $\ell_{1}$ is called canonical if it satisfies the following:

- $g_{t}$ is an isometry on edges and antipodals.

- For any edge $(u, v) \in E\left(G_{t}\right)$, there exists a single coordinate $i$ such that $\left|\left(g_{t}(u)-g_{t}(v)\right)_{i}\right|=1$.

Claim 7. For any integer $t \geq 1$ there is a canonical embedding of $G_{t}$ to $\ell_{1}$.

Proof: By induction on $t$, the base case for $t=0$ is trivial. Assume we have a canonical embedding $g_{t-1}$ : $G_{t-1} \rightarrow \ell_{1}$, and define $g_{t}$ as follows: for every $x \in V\left(G_{t-1}\right)$ let $g_{t}(x)=\bigoplus_{j=1}^{k} g_{t-1}(x)$. It remains to extend $g_{t}$ to the new points. Let $C=C_{2 k}$ be some level $t$ cycle, and note that the antipodal pair $a_{1}, a_{-1}$ is already fixed by $g_{t-1}$. Since $\left(a_{1}, a_{-1}\right) \in E\left(G_{t-1}\right)$ and by the induction hypothesis, there is a single dimension $i$ in which $\mid\left(g_{t-1}\left(a_{1}\right)-g_{t-1}\left(a_{-1}\right)_{i} \mid=1\right.$, so there are $k$ dimensions $I$ in which $\left|\left(g_{t}\left(a_{1}\right)-g_{t}\left(a_{-1}\right)\right)_{i}\right|=1$ for $i \in I$. Denote by $e_{1}, \ldots, e_{k}$ the standard basis unit vectors spanning $I$, and define for each $1 \leq j \leq k-1, g\left(a_{j+1}\right)=g\left(a_{j}\right)+e_{j}$ and $g\left(a_{-(j+1)}\right)=g\left(a_{-j}\right)-e_{j}$.

Note that by the construction of the recursive cycle graph, for any pair $x, y \in V\left(G_{t-1}\right), d_{G_{t}}(x, y)=k \cdot d_{G_{t-1}}(x, y)$, so the edges and antipodals that were preserved by $g_{t-1}$ are also preserved by $g_{t}$. Furthermore, it can be checked that $g_{t}$ is isometry on every level $t$ cycle, and that edges are mapped to one dimension.

\section{A COMbinatorial VieW OF THE BRINKMAN-CHARIKAR PROOF}

In this section we give an alternate view of the Linear Programming proof by Brinkman-Charikar [4] for dimension reduction in $\ell_{1}$ in the large distortion regime using the diamond graphs.

The diamond graphs are constructed as follows. $G_{0}$ consists of a single edge of length 1 . One end point of the edge is designated as the head and the other end point is designated as the tail. $G_{i}$ is constructed from $G_{i-1}$ as follows. For each edge $(s, t)$ in $G_{i-1}$, where $s$ is the head and $t$ is the tail, we replace it with a diamond $s, u, t, v$ with edge length $2^{-i} . s$ is designated as the head of the edges $(s, u)$ and $(s, v) . t$ is designated as the tail of the edges $(u, t)$ and $(v, t)$. The other end points are designated as heads and tails accordingly. The edge $(s, t)$ is called an edge of level $i-1$ and $(v, u)$ is called a diagonal of level $i$.

Now we define some notations with respect to a line embedding $f: G_{t} \rightarrow \mathbb{R}$. Label the original edge in $G_{0}$ the empty string. Consider the diamond in $G_{i}$ corresponding to the edge $(s, t)$ in $G_{i-1}$ with label $x \in\{0,1\}^{i-1}$. Of the two new vertices introduced by the replacement of $(s, t)$ with a diamond, let $u$ be the vertex with smaller $f$ value and $v$ be the vertex with larger $f$ value i.e. $f(u) \leq f(v)$. The difference $f(s)-f(t)$ is called the directed length of the edge $(s, t)$ with head $s$ and tail $t$. The absolute value of the directed length of $(s, t)$ is the length of $(s, t)$. The absolute difference $|f(u)-f(v)|$ is called the diagonal length of the diamond $s, u, t, v$. Label the edges from $s$ to $u$ and from $v$ to $t$ with $x \circ 0$ and label the edges from $s$ to $v$ and from $u$ to $t$ with $x \circ 1$, as shown in Figure 4. Note that for any $x \in\{0,1\}^{i}$, there are $2^{i}$ edges in level $i$ with label $x$. Let $E_{i}$ be the sum of the directed lengths of level $i$ edges, $D_{i}$ be the sum of the lengths of level $i$ diagonals, $E_{i, x}$ be the sum of the directed lengths of level $i$ edges whose labels end with $x$, and $F_{i}$ be the sum of the lengths of the level $i$ edges. Below are some properties relating $E_{i}, D_{i}$, and $E_{i, x}$.

Property 1. $E_{i}+D_{i+1}=E_{i+1,0}$

Proof: Consider an arbitrary diamond in $G_{i+1}$ corresponding to an edge $(s, t)$ in $G_{i}$. Of the two new vertices introduced by the replacement of $(s, t)$ with a diamond, let $u$ be the vertex with smaller $f$ value and $v$ be the vertex with larger $f$ value i.e. $f(u) \leq f(v)$. We have $(f(s)-f(t))+(f(v)-f(u))=(f(s)-f(u))+(f(v)-f(t))$. Summing over all diamonds of $G_{i+1}$, we get the property.

Property 2. $E_{i, x}=\frac{1}{2}\left(E_{i+1, x \circ 0}+E_{i+1, x \circ 1}\right)$. In particular, $E_{i}=\frac{1}{2}\left(E_{i+1,0}+E_{i+1,1}\right)=\frac{1}{2} E_{i+1}$.

Property 3. For any binary string $x$ of length less than $i$, we have $E_{i, x}=E_{i, 0 \circ x}+E_{i, 1 \circ x}$

Theorem 3. Any embedding of $G_{t}$ into $\ell_{1}$ with distortion at most $d$ requires $2^{\Omega\left(t / d^{2}\right)} / d^{2}$ dimensions.

Note that the number of vertices in $G_{t}$ is $n=\Theta\left(4^{t}\right)$ so the theorem states that any embedding of $G_{t}$ into $\ell_{1}$ with distortion at most $d$ requires $\frac{1}{d^{2}} n^{\Omega\left(1 / d^{2}\right)}$ dimensions. Now we get to the proof.

Proof: In order to establish the lower bound, we will prove the following Poincaré-type inequality for any line embedding $f: G_{t} \rightarrow \mathbb{R}$ with stretch at most $s \leq 2^{\Theta\left(t / d^{2}\right)} / d^{2}$,

$$
d \sum_{i=0}^{t-1} 2^{t-i} D_{i+1}-(t-2 d) F_{t} \leq(d+1) \cdot 2^{t}
$$

First, we show the inequality implies the theorem. Apply Lemma 2 with $\beta_{u, v}=d \cdot 2^{t-i}$ for all diagonals $(u, v)$ of level $i+1, \alpha_{s, t}=(t-2 d)$ for all edges $(s, t)$ of level $t$ (set $\beta, \alpha$ zero everywhere else), and $\gamma=(d+1) 2^{t}$. Any embedding with stretch at most $2^{\Theta\left(t / d^{2}\right)} / d^{2}$ has distortion at least 


$$
\begin{aligned}
& \quad \frac{\sum_{x, y \in V\left(G_{t}\right)} \beta_{x, y} d(x, y)}{\sum_{x, y \in V\left(G_{t}\right)} \alpha_{x, y} d(x, y)+\gamma} \\
& =\frac{d \sum_{i=0}^{t-1} 2^{t-i} \cdot 4^{i} \cdot 2^{-i}}{(t-2 d) \cdot 4^{t} \cdot 2^{-t}+(d+1) \cdot 2^{t}}=\frac{d t \cdot 2^{t}}{(t+1-d) 2^{t}} \geq d
\end{aligned}
$$

By Claim 1, the theorem immediately follows.

Now we proceed to prove (8). By Property 1,

$$
\sum_{i=0}^{t-1} 2^{t-i} D_{i+1}=\sum_{i=0}^{t-1} 2^{t-i} E_{i+1,0}-\sum_{i=0}^{t-1} 2^{t-i} E_{i}
$$

By Property 2, $E_{i, x}=\frac{1}{2^{t-i}} \sum_{y \in\{0,1\}^{t-i}} E_{t, x \circ y}$. Apply this identity and Property 3 to the RHS and simplify, we get a sum $\sum_{x \in\{0,1\}^{t}} c_{x} E_{t, x}$ where $c_{x}$ are coefficients depending on $x$. The contribution to $c_{x}$ from $\sum_{i=0}^{t-1} 2^{t-i} E_{i+1,0}$ is $2(t-$ $\left.|x|_{1}\right)$ and from $-\sum_{i=0}^{t-1} 2^{t-i} E_{i}$ is $-t$. Thus, $c_{x}=t-2|x|_{1}$. Let $W=\sum_{x \in\{0,1\}^{t}} m_{x} E_{t, x}$ where

$$
m_{x}= \begin{cases}t-2 d & \text { if }|x|_{1} \leq t / 2-t /(2 d) \\ 2 d-t & \text { if }|x|_{1} \geq t / 2+t /(2 d) \\ d\left(t-2|x|_{1}\right) & \text { otherwise }\end{cases}
$$

We have

$$
\begin{gathered}
d \sum_{i=0}^{t-1} 2^{t-i} D_{i+1}-W=\sum_{x \in\{0,1\}^{t}} E_{t, x}\left(d c_{x}-m_{x}\right) \\
=\sum_{x \in\{0,1\}^{t}:\left.\left|\frac{t}{2}-\right| x\right|_{1} \mid \geq \frac{t}{2 d}} E_{t, x}\left(d\left(t-2|x|_{1}\right)-m_{x}\right)
\end{gathered}
$$

For any $x \in\{0,1\}^{t}$ and $m \in \mathbb{R}$, by the definition of stretch, $m E_{t, x} \leq|m| \cdot\left|E_{t, x}\right| \leq|m| s$, so

$$
\begin{aligned}
& \sum_{x \in\{0,1\}^{t}:\left.\left|\frac{t}{2}-\right| x\right|_{1} \mid \geq \frac{t}{2 d}} E_{t, x}\left(d\left(t-2|x|_{1}\right)-m_{x}\right) \\
& \leq 2 s \sum_{x \in\{0,1\}^{t}:|x|_{1} \leq \frac{t}{2}-\frac{t}{2 d}}\left|d\left(t-2|x|_{1}\right)-(t-2 d)\right| \\
& \leq 2 s \sum_{i=0}^{\frac{t}{2}-\frac{t}{2 d}}\left(\begin{array}{l}
t \\
i
\end{array}\right) \mid\left(d(t-2 i)-t+2 d \mid \leq s d^{2}(1+d) 2^{t-\Theta\left(t / d^{2}\right)}\right.
\end{aligned}
$$

The last inequality follows from a series of calculations in [4]. Notice that $\left|m_{x}\right| \leq t-2 d \forall x$ so $W \leq(t-2 d) F_{t}$. Thus, when the stretch is bounded by $s \leq 2^{\Theta\left(t / d^{2}\right)} / d^{2}$, we have

$$
d \sum_{i=0}^{t-1} 2^{t-i} D_{i+1}-(t-2 d) F_{t} \leq d \sum_{i=0}^{t-1} 2^{t-i} D_{i+1}-W \leq(d+1) 2^{t}
$$

\section{ACKNOWLEDGMENT}

This work was supported by the National Science Foundation under CCF 0832797 and AF 0916218. Part of this work was done while A. A. was a postdoc at the Center for Computational Intractability at Princeton University. H. N. was supported by a Gordon Wu fellowship.

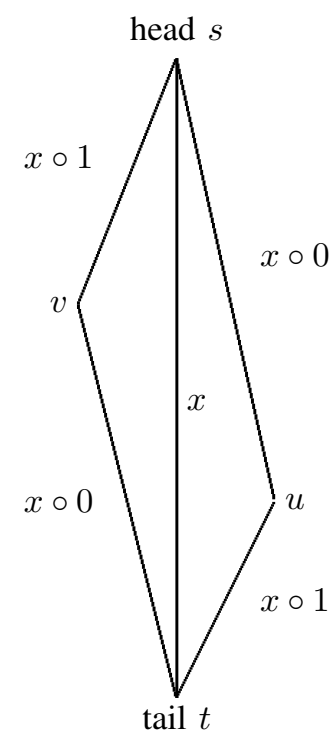

Figure 4. Labels of edges of a diamond $s, u, t, v$ with respect to a line embedding $f$ with $f(u) \leq f(v)$.

\section{REFERENCES}

[1] N. Alon, "Problems and results in extremal combinatorics," I, Discrete Math., vol. 273, pp. 31-53, 2003.

[2] K. Ball, "Markov chains, Riesz transforms and Lipschitz maps," Geom. Funct. Anal., vol. 2, no. 2, pp. 137-172, 1992.

[3] J. D. Batson, D. A. Spielman, and N. Srivastava, "Twiceramanujan sparsifiers," in STOC '09: Proceedings of the 41st annual ACM symposium on Theory of computing. New York, NY, USA: ACM, 2009, pp. 255-262.

[4] B. Brinkman and M. Charikar, "On the impossibility of dimension reduction in $l_{1}$, , J. ACM, vol. 52, pp. 766-788, September 2005. [Online]. Available: http://doi.acm.org/10. 1145/1089023.1089026

[5] M. Charikar and A. Sahai, "Dimension reduction in the $l_{1}$ norm," in FOCS '02: Proceedings of the 43rd Symposium on Foundations of Computer Science. Washington, DC, USA: IEEE Computer Society, 2002, pp. 551-560.

[6] M. Datar, N. Immorlica, P. Indyk, and V. S. Mirrokni, "Locality-sensitive hashing scheme based on p-stable distributions," in SCG '04: Proceedings of the twentieth annual symposium on Computational geometry. New York, NY, USA: ACM, 2004, pp. 253-262.

[7] P. Indyk, "Algorithmic applications of low-distortion geometric embeddings," in Proceedings of the 42nd IEEE symposium on Foundations of Computer Science, ser. FOCS '01. Washington, DC, USA: IEEE Computer Society, 2001, pp. 10-. [Online]. Available: http://portal.acm.org/citation.cfm?id=874063.875596

[8] — "Stable distributions, pseudorandom generators, embeddings, and data stream computation," J. ACM, vol. 53 , no. 3 , pp. 307-323, 2006.

[9] P. Indyk and R. Motwani, "Approximate nearest neighbors: towards removing the curse of dimensionality," in Proceedings of the thirtieth annual ACM symposium on Theory of computing, ser. STOC '98. New York, 
NY, USA: ACM, 1998, pp. 604-613. [Online]. Available: http://doi.acm.org/10.1145/276698.276876

[10] W. B. Johnson and J. Lindenstrauss, "Extensions of Lipschitz mappings into a Hilbert space," in Conference in modern analysis and probability (New Haven, Conn., 1982). Providence, RI: Amer. Math. Soc., 1984, pp. 189-206.

[11] J. M. Kleinberg, "Two algorithms for nearest-neighbor search in high dimensions," in Proceedings of the twenty-ninth annual ACM symposium on Theory of computing, ser. STOC '97. New York, NY, USA: ACM, 1997, pp. 599608. [Online]. Available: http://doi.acm.org/10.1145/258533. 258653

[12] E. Kushilevitz, R. Ostrovsky, and Y. Rabani, "Efficient search for approximate nearest neighbor in high dimensional spaces," SIAM Journal on Computing, vol. 30, no. 2, pp. 457-474, 2000. [Online]. Available: http://link.aip.org/link/ ?SMJ/30/457/1

[13] J. Lee and A. Naor, "Embedding the diamond graph in $l_{p}$ and dimension reduction in $l_{1}$," Geometric and Functional Analysis (GAFA) 14(4), pp. 745-747, 2004.

[14] I. Newman and Y. Rabinovich, "On cut dimension of $l_{1}$ metrics and volumes, and related sparsification techniques," in arxiv: $\quad$ arxiv.org/abs/1002.3541, 2010.

[15] G. Schechtman, "More on embedding subspaces of $l_{p}$ in $l_{r}^{n}$," Composio Math., vol. 61(2), pp. 159-169, 1987.

[16] M. Talagrand, "Embedding subspaces of $l_{1}$ into $l_{1}^{n}$," Proceedings of the American Mathematical Society, vol. 108, no. 2, pp. pp. 363-369, 1990. [Online]. Available: http://www.jstor.org/stable/2048283 